1 2

Journal of Computational Neuroscience manuscript No. (will be inserted by the editor)

\title{
A simple integrative electrophysiological model of bursting GnRH neurons
}

\author{
Dávid Csercsik · Imre Farkas · Erik Hrabovszky · Zsolt Liposits
}

Received: date / Accepted: date

Abstract In this paper a modular model of the GnRH neuron is presented. For the aim of simplicity, the currents corresponding to fast time scales and action potential generation are described by an impulsive system, while the slower currents and calcium dynamics are described by usual ordinary differential equations (ODEs). The model is able to reproduce the depolarizing afterpotentials, afterhyperpolarization, periodic bursting behavior and the corresponding calcium transients observed in the case of GnRH neurons.

\begin{abstract}
This work was supported by the National Science Foundation of Hungary K69127, K67625, and T73002) and the Hungarian Health Research Council Fund (122/2009). The research leading to these results has received funding from the European Community's Seventh Framework Program (FP7/ 2007-2013) under Grant Agreement 245009. The authors would like to thank Katalin Hangos for her contribution.
\end{abstract}

Dávid Csercsik $^{1} \cdot$ Imre Farkas $^{2} \cdot$ Erik Hrabovszky $^{2} \cdot$ Zsolt Liposits $^{2,3}$

${ }^{1}$ Process Control Research Group

Computer and Automation Research Institute

Hungarian Academy of Sciences

H-1518, P.O. Box 63, Budapest, Hungary

Tel.: +3612796163

Fax: +3614667503

E-mail: \{csercsik\}@scl.sztaki.hu

${ }^{2}$ Department of Endocrine Neurobiology

Institute of Experimental Medicine,

Hungarian Academy of Sciences

H-1450 Budapest, P.O. Box 67, Hungary

Tel: +36 12109943

Fax: +3612109944

${ }^{3}$ Faculty of Information Technology Pázmány Péter Catholic University, H-1364 Budapest 4., P.O. Box 178 , Hungary Tel: +3618864700

Fax: +3618864724

\section{Introduction}

Gonadotropin-releasing hormone $(\mathrm{GnRH})$ is a fundamental central element in the regulation of the reproductive system in vertebrates. GnRH is secreted by GnRH neurons, hypothalamic neuroendocrine cells, the activity of which is controlled by a neuronal pulse generator possibly located in the arcuate nucleus (Navarro et al., 2009; Lehman et al., 2010). The pulsatile release of GnRH, which is closely associated with concurrent increases in multiunit electrical activity in the mediobasal hypothalamus (Knobil, 1980; Conn \& Freeman, 2000), is driven by the activity of GnRH neurons, characterized by bursts and prolonged episodes of repetitive action potentials (APs). The correlation of action potential bursts with oscillatory increases in intracellular $\mathrm{Ca}^{2+}$ has been shown in the case of GT1 cells (hypothalamic GnRH neurons immortalized by genetically targeted tumorigenesis) (Constantin \& Charles, 1999) as well as in the case of green fluorescent protein (GFP) tagged GnRH neurons (Spergel et al., 1999) from hypothalamic slices (Lee et al., 2010).

Several in vitro experiments have shown, that the secretory activity of GnRH cells is determined by changes in cytosolic $\mathrm{Ca}^{2+}$ concentration (Stojilkovic et al., 1994), which plays a central role in the signal transduction processes that lead to exocytosis. GnRH secretion from perifused GT1 and hypothalamic cells is reduced by L-type $\mathrm{Ca}^{2+}$ channel inhibitors and augmented by activation of voltage-gated $\mathrm{Ca}^{2+}$ channels (Krsmanovic et al., 1992).

A couple of models have been constructed so far to describe the ionic currents and short term (considering scales of milliseconds) general electrophysiological (Csercsik et al., 2010), and dendritic properties (Roberts et al., 2006; Roberts, Hemond, \& Suter, 2008; Roberts et al., 2009) of GnRH neurons based on measurements performed on cells from hypothalamic slices. Other mathematical models (LeBeau 
et al., 2000; Van Goor et al., 2000; Fletcher \& Li, 2009) have been proposed to explain experimental data obtained on GT1 cells. These models focus mainly on the autocrine regulation by GnRH through adenylyl cyclase and calcium coupled pathways.

Bursting behavior of GnRH neurons (Kuehl-Kovarik et al., 2005), which is likely connected to cytosolic $\mathrm{Ca}^{2+}$ transients and changes when endocrine status is altered, has been in the focus of interest in the past years. The model described in (Fletcher \& Li, 2009), which is based on data originating from GT1 cell lines, incorporates an apamin sensitive channel, which is able to terminate bursting behavior during IP3 gated $\mathrm{Ca}^{2+}$ transients. This model also assumes a "store operated" calcium current. The mechanism of apamin sensitive hyperpolarization has been verified also in the case of hypothalamic cells (Lee et al., 2010). However the bursting patterns, and the corresponding $\mathrm{Ca}^{2+}$ transients are significantly different in the case of hypothalamic neurons (as it is described in (Lee et al., 2010), in most of the time - between bursts - neither calcium transients, nor action currents corresponding to APs appear).

A recently published paper (Lee et al., 2010), which is based on simultaneous loose-patch recordings and calcium imaging, aims the description of bursting behavior via $\mathrm{Ca}^{2+}$ dependent potassium currents. The bifurcation analysis of the model can be found in (Duan et al., 2011). The loosepatch recording measurement method can be considered as the one influencing the cell's interior $\mathrm{Ca}^{2+}$ dynamics at least. In addition the authors form a mathematical model to demonstrate that $\mathrm{Ca}^{2+}$ dependent potassium currents may control the bursting behavior. The aims of our present work are to simplify this model and also to simultaneously incorporate one active and one passive dendritic compartment (to make it able to describe dendritic AP generation (Roberts, Hemond, \& Suter, 2008) in bipolar GnRH neurons), and a current corresponding to a possible mechanism which has not been taken into account in the original model (Lee et al., 2010). This mechanism is the assumed $\mathrm{Ca}^{2+}$ dependent regulation of sodium channels which, as we will show later, makes the model able to explain depolarizing afterpotentials (DAPs or afterdepolarizations ADPs). DAPs are considered an important characteristic feature of the electrophysiological behavior of GnRH neurons (Chu \& Moenter, 2006), which contribute to bursting behavior (Kuehl-Kovarik et al., 2005). The simplification of the model framework takes place via the application of the 'simple model' described in (Izhikevich, 2005). This approach has the benefit, that it can describe subthreshold behavior and the depolarizing phase of AP generation with only an amplifying and a resonant variable, which is the minimal model of excitability. In addition, the number of parameters is significantly lower compared to the case of a detailed conventional Hodgkin-Huxley type model (Csercsik et al., 2010). The tradeoff is that we are not able to handle the appearing voltage gated currents separately (except for the calcium current, as we will see, which has a leading role in the model).

\subsection{Characteristic features to be described by the model}

The above mentioned experimental observations indicate important characteristic features of GnRH neurons, which should be reproduced by the model. In particular, these are as follows.

1. The model should be able to approximately reproduce the shape of action potentials (APs), and excitability properties observed in GnRH neurons originating from hypothalamic slices (Sim et al., 2001; Chu \& Moenter, 2006; DeFazio \& Moenter, 2002; Csercsik et al., 2010). Furthermore, the model should be able to reproduce dendritic AP generation (Roberts, Campbell, et al., 2008).

2. The model should be able to describe DAPs (KuehlKovarik et al., 2005; Chu \& Moenter, 2006), because we suppose that DAPs significantly contribute to bursting behavior (Kuehl-Kovarik et al., 2005).

3. The model should reproduce long lasting hyperpolarizing currents and afterhyperpolarization (AHP), regulating interburst intervals (Lee et al., 2010).

4. The model should be able to reproduce typical bursts observed in GnRH neurons (Kuehl-Kovarik et al., 2005; Lee et al., 2010), and the corresponding calcium transients (Lee et al., 2010).

If we consider that APs appear on the scale of milliseconds, while DAPs and periodic bursting behavior can be observed in the scale of seconds and tens of seconds, we have to note that the above requirements imply that the model should perform acceptable on different time scales. In addition, the mathematical implementation has to be as simple as possible for the model to remain computationally feasible.

\section{Electrophysiology}

In the following subsections the electrophysiological methodology and measurements are described.

\subsection{Animals}

Adult, male GnRH-green-fluorescent protein (GnRH-GFP) transgenic mice (kind gift of Dr. Suzanne Moenter) bred on a C57B1/6J genetic background were used for the electrophysiological experiments. In this animal model, a GnRH promoter segment drives selective GFP expression in the majority of GnRH neurons (Suter et al., 2000). The animals were housed in light (12:12 light-dark cycle, lights on at 07:00h)- and temperature $\left(22 \pm 2^{\circ} \mathrm{C}\right)$ controlled environment, with free access to standard food and tap water. All studies were carried out with the permissions of the Animal Welfare Committee of the Institute of Experimental Medicine Hungarian Academy of Sciences (No.: A5769-01) 
and were in accordance with legal requirements of the European Community (Decree 86/609/EEC).

\subsection{Brain slice preparation and recording}

Brain slices were prepared as described earlier (Farkas et al., 2010). Briefly, mice were killed by cervical dislocation. The brain was removed rapidly and immersed in ice cold artificial cerebrospinal fluid (aCSF; in mM: $\mathrm{NaCl} 135, \mathrm{KCl} 3.5, \mathrm{NaHCO}_{3} 26, \mathrm{MgSO}_{4} 1.2, \mathrm{NaH}_{2} \mathrm{PO}_{4}$ $1.25, \mathrm{CaCl}_{2} 2.5$, glucose 10) which had been bubbled with a mixture of $95 \% \mathrm{O}_{2}$ and $5 \% \mathrm{CO}_{2}$. Hypothalamic blocks were dissected and 300 $\mu \mathrm{m}$ (for whole cell patch clamp) or $450 \mu \mathrm{m}$ (for loose-patch recordings) thick coronal slices were prepared at the level of the medial septum/preoptic area with a vibratome in ice-cold oxygenated aCSF. The slices containing the preoptic area were bisected along the midline and equilibrated in aCSF saturated with $\mathrm{O}_{2} / \mathrm{CO}_{2}$ at room temperature for 1 h. During recording, the brain slices were oxygenated by bubbling the aCSF with $\mathrm{O}_{2} / \mathrm{CO}_{2}$ gas at $33^{\circ} \mathrm{C}$. Axopatch $200 \mathrm{~B}$ patch-clamp amplifier, Digidata-1322A data acquisition system, and pCLAMP 9.2 software (Molecular Devices Co., USA) were used for recording. GnRHGFP neurons were identified by brief illumination at $470 \mathrm{~nm}$ using an epifluorescent filter set, based on their green fluorescence (Suter et al., 2000)

\subsubsection{Morphology of GnRH neurons}

As described in (Campbell et al., 2005), biocytin filling experiments in GnRH neurons revealed that majority (about 65\%) of GnRH neurons exhibit a bipolar morphology. Regarding our measurements, while the typical bipolar morphology of GnRH-GFP neurons was also obvious in most of our recorded cells, in other cases it was not possible to determine cell morphology in the absence of biocytin filling.

\subsection{Whole-cell recording of GnRH neurons}

Slices were transferred to the recording chamber, held submerged, and continuously superfused with oxygenized aCSF. All recordings were made at $33^{\circ} \mathrm{C}$.

GnRH-GFP neurons were identified in the acute brain slices by their green fluorescence.

The electrodes were filled with intracellular solution (in mM): 140 $\mathrm{KCl}, 10 \mathrm{HEPES}, 5$ EGTA, $0.1 \mathrm{CaCl}_{2}, 4 \mathrm{MgATP}, 0.4 \mathrm{NaATP}$, pH 7.3 with $\mathrm{NaOH}$. Resistance of patch electrodes was 2-3 M $\Omega$. Holding potential was $-70 \mathrm{mV}$, near the average resting potential of the GnRH cells. Pipette offset potential, series resistance and capacitance were compensated before recording.

\subsubsection{Recording of action potentials}

Current clamp measurements were performed on 5 cells with depolarizing current steps of various amplitudes. The holding current was 0 pA. The injected current (30 pA, $200 \mathrm{~ms}$ ) was appropriate to evoke APs in most of the cells. The $30 \mathrm{pA}$ traces of the cells are depicted in Fig. 1.

\subsubsection{Recording of spontaneous postsynaptic currents ( $s$ PSCs)}

The cells were voltage-clamped at a holding potential of $-70 \mathrm{mV}$. The recorded postsynaptic current traces are depicted in Fig. 1.
As it is described in (Farkas et al., 2010), the postsynaptic currents measured in this configuration correspond to GABAa receptor activation, which, although there are some controversial results (Han et al., 2002, 2004), is assumed to affect adult GnRH neurons in an excitatory way (DeFazio et al., 2002; Moenter \& DeFazio, 2005; Watanabe et al., 2009; Yin et al., 2008). Although there are other types of (mainly glutamate mediated) synaptic inputs to GnRH neurons (Suter, 2004; Kuehl-Kovarik et al., 2002), measurements of postsynaptic currents in this configuration usually fail to detect these glutamatergic excitatory currents. Partly, this can be related to the technical issue, that glutamatergic input is often received by distal dendrites, which may exert lower effect on the recordings from cell bodies.

\subsection{Loose-patch-clamp experiments}

In loose-patch method, the recording pipette is attached loosely to the extracellular surface of the cell membrane and thus the composition of the pipette solution is similar to that of the extracellular solution. We were interested in the spontaneous firing of the cell, therefore, the pipette potential was set to $0 \mathrm{mV}$ the potential of the extracellular solution. Using loose-patch method, electrical discharges called action currents were recorded. Action current is the membrane current associated with action potential firing.

Recording of action current firing of GnRH neurons was carried out as described earlier (Farkas et al., 2010). Briefly, recordings were carried out in aCSF bubbled with $\mathrm{O}_{2} / \mathrm{CO}_{2}$ gas at $33^{\circ} \mathrm{C}$. Pipette potential was $0 \mathrm{mV}$, pipette resistance $1-2 \mathrm{M} \Omega$, resistance of loose-patch seal 7-40 $\mathrm{M} \Omega$. Composition of the pipette solution was the following (in $\mathrm{mM}$ ): $\mathrm{NaCl} 150, \mathrm{KCl} 3.5, \mathrm{CaCl}_{2} 2.5, \mathrm{MgCl}_{2}$ 1.3, HEPES 10, glucose $10(\mathrm{pH}=7.3$ with $\mathrm{NaOH})$. The recorded action current traces, on which the burst firing patterns can be clearly seen, are shown in Fig. 1.

Analysis of burst firing revealed a mean burst duration of $2.5 \pm 0.9$ $\mathrm{s}$, mean spikes-per-burst of $6 \pm 3$, mean interburst interval of $16.4 \pm 7$ $\mathrm{s}$, mean interspike interval of $519 \pm 182 \mathrm{~ms}$, and a mean number of $1.3 \pm 1.26$ single (not belonging to any bursts) spikes. During the analysis, neighboring spikes were considered to belonging to the same burst if they were less then $1.5 \mathrm{~s}$ apart from one another. These values are in good agreement with the statistical data published in (Lee et al., 2010) regarding loose-patch experiments.

\section{Model description}

In the following subsections the modelling assumptions and the implied model equations are described. The model parameters can be found in the appendix.

\subsection{Membrane voltage sub-model}

Considering earlier results that underline the importance of dendritic mechanisms in the case of GnRH neurons (Campbell et al., 2009; Roberts et al., 2006; Roberts, Hemond, \& Suter, 2008) which may contribute to the description of AP generation (Roberts, Campbell, et al., 2008), bursting (Izhikevich, 2005) and DAPs (Roberts et al., 2009), we take into account an active dendritic compartment. Furthermore, because the majority of our measurement data originates from bipolar GnRH neurons, a second, passive dendritic compartment is also incorporated in the model. This will allow us to study the somato-dendritic interactions in the model, without the needs for more complex spatial models and morphological considerations (see eg. (Roberts et al., 2009)). In contrast 
a

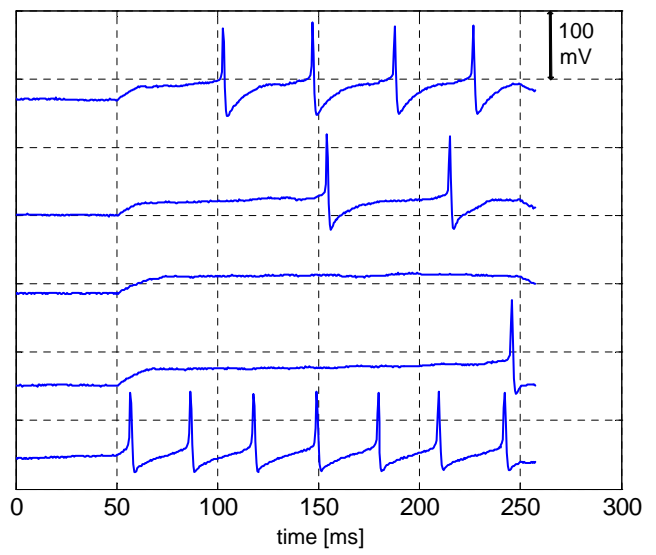

b

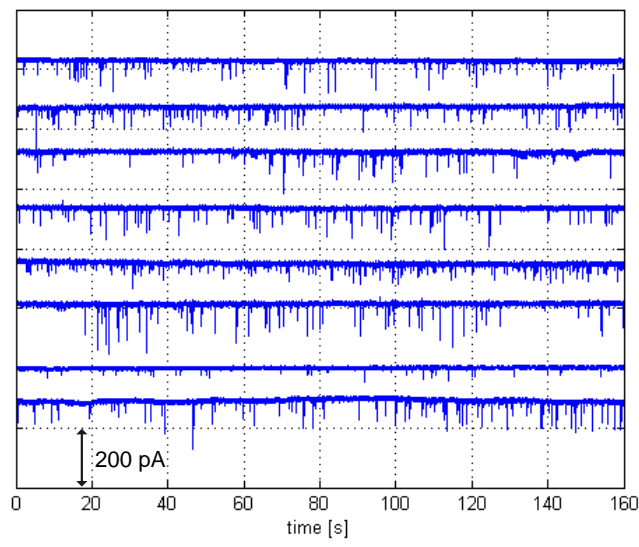

\section{C}

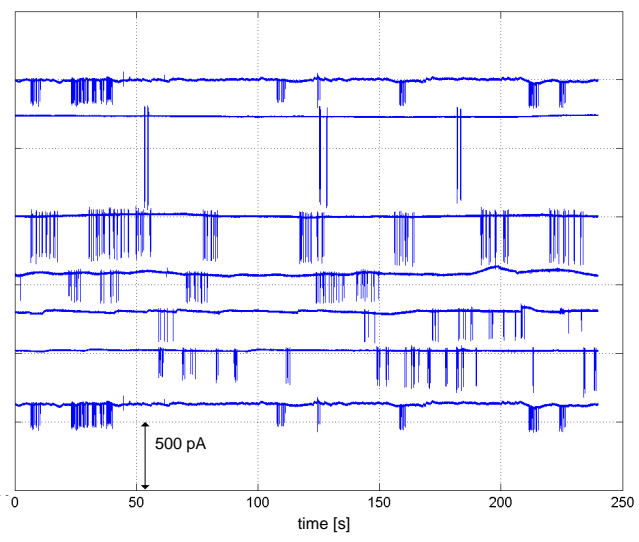

Fig. 1 Electrophysiological measurement results a) The $30 \mathrm{pA}$ current clamp (CC) traces of 5 different cells. The resting potential values of the cells were: $-71,-69.2,-73,-79$, and $-53 \mathrm{mV}$, from top to bottom. The mean number of APs was 2.8. b) GABA mediated postsynaptic currents of 8 different cells measured at a holding potential of $-70 \mathrm{mV}$. c) Traces of action currents from $7 \mathrm{GnRH}-\mathrm{GFP}$ neurons revealing burst firing in loose-patch configuration.

to the models described earlier (Csercsik et al., 2010; Lee et al., 2010; Roberts et al., 2009), we do not describe the voltage dependent ionic currents separately via the modu- lar Hodgkin-Huxley framework, but we use an integrated approach. Based on (Izhikevich, 2005), to describe action potential generation, we use the following impulsive system (Bainov \& Simeonov, 1989) for the characterization of the membrane potential and voltage gated inactivation currents for the somatic compartment

$$
\begin{aligned}
& C \dot{v}= k\left(v-v_{r}\right)\left(v-v_{t}\right)+c_{s}\left(v^{d}-v\right)+ \\
&+c_{p s}\left(v^{p d}-v\right)-u+\left(1-\left(r_{d}+r_{p d}\right)\right) \sum I+I_{\text {inj }}^{s} \\
& \dot{u}= a\left(b\left(v-v_{r}\right)-u\right) \\
& \text { if } \quad v \geq v_{\text {peak }}, \text { then } v \leftarrow c, u \leftarrow u+d
\end{aligned}
$$

For the active and passive dendritic compartments, the equations are:

$$
\begin{aligned}
C^{d} \dot{v}^{d} & =k^{d}\left(v^{d}-v_{r}^{d}\right)\left(v^{d}-v_{t}^{d}\right)+c_{d}\left(v-v^{d}\right)-u^{d}+r_{d} \sum I+I_{i n j}^{d} \\
u^{d} & =a^{d}\left(b^{d}\left(v^{d}-v_{r}^{d}\right)-u^{d}\right) \\
& \text { if } v^{d} \geq v_{p e a k}^{d}, \text { then } v^{d} \leftarrow c^{d}, u^{d} \leftarrow u^{d}+d^{d} \\
C^{p d} \dot{v}^{p d} & =c_{p d}\left(v-v^{d}\right)+r_{p d} \sum I+I_{i n j}^{p d}
\end{aligned}
$$

where $\mathrm{C}$ is the capacitance $[\mathrm{pF}], v[\mathrm{mV}]$ denotes the (somatic) membrane potential, $u$ [pA] is used for the notation of voltage-gated inactivation currents. $v_{r}[\mathrm{mV}]$ and $v_{t}[\mathrm{mV}]$ denote the resting and threshold potentials, respectively. In addition to $v_{r}$ and $v_{t}$, the parameters $k>0, a>0$ and $b>0$ $[\mathrm{pA} / \mathrm{mV}]$ correspond to the geometry of nullclines in the neighborhood of the resting state (as described in (Izhikevich, 2005)). The upper index ${ }^{d}$ in general refers to the same quantities in the case of the active dendritic compartment. The voltage and capacitance of the passive dendritic compartment are denoted with the upper index ${ }^{p d} . c_{s}>0$ and $c_{d}>0$ $[\mathrm{pa} / \mathrm{mV}]$ correspond to the coupling between the somatic and the active dendritic compartment, and $c_{p s}>0$ and $c_{p d}>$ 0 describe the coupling between the soma and the passive dendrite. $c[\mathrm{mV}]$, and $d>0[\mathrm{pA}]$ are the parameters of the voltage triggered impulsive effect (voltage reset). $\sum I$ corresponds to the sum of calcium dependent currents (which are described later). As it is described in the review article (Verkhratsky, 2005), the endoplasmic reticulum (ER), which significantly contributes to $\mathrm{Ca}^{2+}$ transients, extends from the nucleus and the soma to the dendritic arborization and, through the axon, even to presynaptic terminals. According to this, we will assume that a fraction of $0<\left(r_{d}+r_{p d}\right)<1$ of $\mathrm{Ca}^{2+}$-dependent currents appear in the dendritic compartments, whereas the rest $\left(1-\left(r_{d}+r_{p d}\right)\right)$ flows in the soma. Additional currents injected to the somatic or to the dendritic compartment are described by $I_{i n j}^{s}, I_{i n j}^{d}, I_{i n j}^{p d}$.

\subsection{Voltage dependent calcium currents}

As stated before, we do not take into account all of the different voltage dependent currents separately. However, voltage dependent $\mathrm{Ca}^{2+}$ currents have significant importance in the model, because they basically contribute to the intracellular $\mathrm{Ca}^{2+}$ dynamics. It is known that many subtypes of voltage dependent $\mathrm{Ca}^{2+}$ channels can be found in $\mathrm{GnRH}$ neurons 
(see eg. (Kato et al., 2003; Herbison et al., 2001; Watanabe et al., 2004; Krsmanovic et al., 1992; Nunemaker et al., 2003)). Although these channels exhibit different dynamical features, to keep the model as simple as possible, we try to describe their overall contribution with single HodgkinHuxley type ion channel model.

We will assume, that the effect of voltage dependent currents on the membrane potential is included in eq. 1, which means that we will not take them into account separately in the equations corresponding to the membrane voltage submodel. They will describe how the voltage submodel affects the calcium submodel. For the aim of simplicity, we will take into account voltage dependent $\mathrm{Ca}^{2+}$ currents only in the somatic compartment. If we wanted to take into account $\mathrm{Ca}^{2+}$ currents also in the dendritic compartments, $\mathrm{Ca}^{2+}$ concentrations, and the corresponding dynamics of ER and IP3 signaling would have to be modelled in the dendrites also, which would mean several further state variables, and parameters. The equations describing the dynamics of the voltage dependent $\mathrm{Ca}^{2+}$ currents are the following

$I_{C a}=g_{C a} m_{C a}^{2} h_{C a}^{2}\left(v-E_{C a}\right)$
$\dot{m}_{C a}=\frac{m_{C a}^{\infty}-m_{C a}}{\tau_{C a}^{m}} \quad \dot{h}_{C a}=\frac{h_{C a}^{\infty}-h_{C a}}{\tau_{C a}^{h}}$

where the steady-state values $\left(m_{C a \infty}\right.$ and $\left.h_{C a \infty}\right)$ and voltage dependent time constants $\left(\tau_{C a}^{m}\right.$ and $\tau_{C a}^{h}$ ) are described by Boltzmann and Gauss type functions, as usual.

$$
\begin{aligned}
m_{C a}^{\infty} & =\frac{1}{1+e^{\frac{V_{1 / 2 m C a}-v}{K_{m C a}}}} \quad h_{C a}^{\infty}=\frac{1}{1+e^{\frac{V_{1 / 2 h C a}-v}{K_{h C a}}}} \\
\tau_{C a}^{m} & =C_{b a s e}^{m C a}+C_{a m p}^{m C a} \exp \left(\frac{-\left(V_{\max m C a}-v\right)^{2}}{\sigma_{m C a}^{2}}\right) \\
\tau_{C a}^{h} & =C_{b a s e}^{h C a}+C_{a m p}^{h C a} \exp \left(\frac{-\left(V_{\max } h C a-v\right)^{2}}{\sigma_{h C a}^{2}}\right)
\end{aligned}
$$

As we can see, the voltage dependent transmembrane $\mathrm{Ca}^{2+}$ currents depend on the membrane potential of the soma.

The reversal potential of $\mathrm{Ca}^{2+}$ is described via the Nernst equation

$$
E_{C a}=31 \log _{10}\left(c_{\text {ext }} / c\right)
$$

where $c_{\text {ext }}$ is the external $\mathrm{Ca}^{2+}$ concentration, which is assumed to be constant, and $c$ is the concentration of $\mathrm{Ca}^{2+}$ in the cytosol.

\subsection{Calcium sub-model}

GnRH neurons display $\mathrm{Ca}^{2+}$ transients, driven primarily by their burst firing (Jasoni et al., 2010).

If the calcium, which enters the cell via voltage dependent $\mathrm{Ca}^{2+}$ currents, exceeds a certain concentration threshold, it may trigger an inositol-triphosphate (IP3) dependent $\mathrm{Ca}^{2+}$ transient (Finch et al., 1991), which is related to the IP3 gated efflux of $\mathrm{Ca}^{2+}$ from the endoplasmic reticulum (ER) to the cytosol. This means that the $\mathrm{Ca}^{2+}$ submodel of the system is also excitable. To model the mechanisms which ensure this important property, we take two $\mathrm{Ca}^{2+}$ compartments into account, one corresponding to the cytosol, and one corresponding to the ER as proposed earlier (Lee et al., 2010).

We assume that the calcium dynamics are influenced by the $\mathrm{Ca}^{2+}$ currents of the somatic compartment (as described in 3.2, the voltage dependent $\mathrm{Ca}^{2+}$ currents are taken into account only in this compartment). The assumed calcium fluxes between the cytosol, the endoplasmic reticulum (ER), and the extracellular space are summarized in Fig. 2. Considering the calcium submodel, we mainly rely on the model described in (Lee et al., 2010).

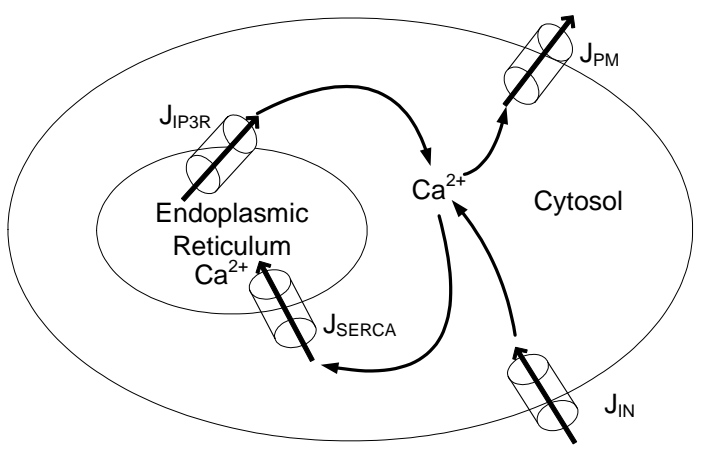

Fig. 2 Calcium fluxes of the model.

According to the $\mathrm{Ca}^{2+}$ fluxes defined in Fig. 2, the differential equations describing the $\mathrm{Ca}^{2+}$ concentration in the cytosol $(c[\mu \mathrm{M}])$ and in the endoplasmic reticulum $\left(c_{e}[\mu \mathrm{M}]\right)$ will take the form

$\dot{c}=J_{I P 3 R}-J_{S E R C A}+\rho\left(J_{I N}-J_{P M}\right) \quad \dot{c}_{e}=\gamma\left(J_{S E R C A}-J_{I P 3 R}\right)$

where $\rho$ and $\gamma$ correspond to the volume ratio of the various compartments. The fluxes are the following:

- $J_{I P 3 R}\left[\mu \mathrm{M} \mathrm{ms}^{-1}\right]$ denotes the $\mathrm{Ca}^{2+}$ flux from the endoplasmic reticulum (ER) to the cytosol via inositol 1,4,5triphosphate (IP3) gated $\mathrm{Ca}^{2+}$ channels. In addition to the intracellular messenger IP3, the functioning of these channels is regulated also by cytosolic calcium. We suppose that each functional unit of the homotetramer IP3 receptor (IP3R) on the membrane of the ER is composed of an IP3-binding activation domain, a $\mathrm{Ca}^{2+}$-binding activation domain, and $\mathrm{Ca}^{2+}$-binding inactivation domain. It is assumed that for the opening of the channel, at least three of the four such units have to be in the activated state. This will cause $\mathrm{Ca}^{2+}$ to enhance the IP3R gated channel opening at lower concentrations, and inhibit it at high concentrations (Bezprozvanny et al., 1991; Finch et al., 1991; Parker \& Ivorra, 1990). According to these assumptions, and considering the model described in $(\mathrm{Li}$ $\&$ Rinzel, 2010), $J_{I P 3 R}$ takes the following form:

$J_{I P 3 R}=\left(K_{f}\left(\left(\frac{I P 3}{I P 3+K_{i}}\right)\left(\frac{c}{c+K_{a}}\right) y\right)^{3}+J_{e r}\right)\left(c_{e}-c\right)$

where $K_{f}>0\left[\mathrm{~ms}^{-1}\right]$ is the maximal conductance of the IP3 coupled current, IP3 is the IP3 concentration $[\mu \mathrm{M}]$, 
$K_{i}>0[\mu \mathrm{M}]$ is the dissociation constant corresponding to IP3 and the IP3-binding activation-domain of the IP3R. $K_{a}[\mu \mathrm{M}]$ is the dissociation constant corresponding to $\mathrm{Ca}^{2+}$ and the $\mathrm{Ca}^{2+}$-binding activation-domain of the IP3R. $y$ is the ratio of the free (unbound) $\mathrm{Ca}^{2+}$-binding inactivation domains of IP3R, described by the differential equation: $\dot{y}=A\left(K_{d}(1-y)-c y\right)$, where $K_{d}>0[\mu \mathrm{M}]$ is the dissociation constant corresponding to $\mathrm{Ca}^{2+}$ and the $\mathrm{Ca}^{2+}$-binding inactivation-domain of the IP3R and $A>0\left[\mu \mathrm{M}^{-1} \mathrm{~ms}^{-1}\right]$ is constant. At this initial state of model development, we suppose the IP3 concentration to be constant. Later the model can be extended with the description of phospholipase-C dependent signaling mechanisms (for eg. GnRH autoregulation), which influence this value.

- J JERCA $\left[\mu \mathrm{M} \mathrm{ms}^{-1}\right]$ denotes the calcium flux related to the sarco/endoplasmic reticulum $\mathrm{Ca}^{2+}$-ATPase, which pumps the $\mathrm{Ca}^{2+}$ from the cytosol to the ER, opposite to the driving force of the concentration gradient (physiological $\left[\mathrm{Ca}^{2+}\right]$ in the cytosol is about $0.05-0.2 \mu \mathrm{M}$, a few hundred $\mu \mathrm{M}$ in the $\mathrm{ER}$, and few $\mathrm{mM}$ in the extracellular medium).

$J_{S E R C A}=P_{\text {rate }} \frac{c-a_{1} c_{e}}{a_{2}+a_{3} c+a_{4} c_{e}+a_{5} c c_{e}}$

where the positive parameters $P_{\text {rate }}, a_{1}$ (which are dimensionless) and $a_{2}, a_{3}, a_{4}[\mathrm{~ms}]$ and $a_{5}[\mathrm{~ms} / \mu M]$ describe the dependence of pumping efficiency on cytosolic and $\mathrm{ER}\left[\mathrm{Ca}^{2+}\right]$.

- $J_{I N}\left[\mu \mathrm{M} \mathrm{ms}^{-1}\right]$ denotes the $\mathrm{Ca}^{2+}$ flux from outside to inside of the cell. $J_{I N}=-\alpha I_{C a}$ where the constant $\alpha$ $\left[\mu \mathrm{M} \mathrm{ms}^{-1} \mathrm{pA}^{-1}\right]$ corresponds to the cell volume, and describes how much the cytosolic calcium concentration is influenced by the transmembrane current. $I_{C a}[\mathrm{pA}]$ denotes the voltage dependent $\mathrm{Ca}^{2+}$ current described by the equation 3.

- $J_{P M}\left[\mu \mathrm{M} \mathrm{ms}^{-1}\right]$ denotes the active $\mathrm{Ca}^{2+}$ transport (opposite to the driving force of the concentration gradient) through the plasma membrane. It consists of two terms, one corresponding to the $\mathrm{Ca}^{2+}$ ATPase, and the other corresponding tho the $\mathrm{Na}^{+}-\mathrm{Ca}^{2+}$ exchanger

$J_{P M}=V_{P} \frac{c^{2}}{c^{2}+K_{P}^{2}}+V_{N a C a} \frac{c^{4}}{c^{4}+K_{N a C a}^{4}}$

where $V_{P}\left[\mu \mathrm{M} \mathrm{ms}^{-1}\right], K_{P}[\mu \mathrm{M}], V_{N a C a}\left[\mu \mathrm{M} \mathrm{ms}^{-1}\right]$ and $K_{\mathrm{NaCa}}[\mu \mathrm{M}]$ are the positive parameters of this flux.

\subsection{Calcium dependent currents}

As it was foreshadowed, the term $\sum I$ in equation 1 stands for the $\mathrm{Ca}^{2+}$ dependent currents. In addition to the voltage dependent calcium current $I_{C a}$, these currents mean the connection between the voltage and the calcium submodel. The $\mathrm{Ca}^{2+}$ dependent currents are as follows.

\subsection{1 $I_{S K}$}

$I_{S K}$ denotes the fast activating, apamin-sensitive potassium current, which mediates medium and long duration AHPs. Based on (Lee et al., 2010), the equation of this current is

$I_{S K}=g_{S K} \frac{c^{3}}{c^{3}+K_{S K}^{3}}\left(v-E_{K}\right)$

where $g_{S K}[\mathrm{nS}]$ is the maximum conductance, $K_{S K}[\mu \mathrm{M}]$ describes the $\mathrm{Ca}^{2+}$ dependence, and $E_{K}[\mathrm{mV}]$ is the constant equilibrium potential of potassium. As we can see, this current owns no dynamical variables, its value can be explicitly expressed from the actual cytosolic $\mathrm{Ca}^{2+}$ level.

\subsection{2 $I_{U C L}$}

$I_{U C L}$ denotes the UCL2077-sensitive slow activating potassium current, responsible for long duration AHP-s. The equation taken from (Lee et al., 2010) is

$I_{U C L}=g_{U C L}\left(O_{U C L}+O_{U C L}^{*}\right)\left(v-E_{K}\right)$

where $O_{U C L}$ and $\mathrm{O}_{U C L}^{*}$ (corresponding to open states of the channel) are governed by the kinetic equations of the system depicted in (Lee et al., 2010).

$$
\begin{aligned}
& \frac{d S_{U C L}}{d t}=-c k_{1}^{+} S_{U C L}+k_{1}^{-} O_{U C L}+k_{3}^{+} O_{U C L}^{*} \\
& \frac{d O_{U C L}}{d t}=c k_{1}^{+} S_{U C L}-k_{1}^{-} O_{U C L}-k_{2}^{+} O_{U C L} \\
& \frac{d O_{U C L}^{*}}{d t}=k_{2}^{+} O_{U C L}-k_{3}^{+} O_{U C L}^{*}
\end{aligned}
$$

\subsection{3 $I_{D A P}$}

It has been shown (Chu \& Moenter, 2006) that DAPs, which contribute to bursting (Kuehl-Kovarik et al., 2005), observed in the case of GnRH neurons are TTX dependent. Furthermore, in (Chu \& Moenter, 2006) it has been shown, that the broadspectrum calcium channel blocker cadmium reduced the amplitude of DAP by $60 \%$, although it has not reduced the amplitude of the corresponding current $I_{D A P}$. The authors raise the possibility that this influence of DAP amplitude is caused through the modulation of calcium activated potassium currents. However, it can be speculated that the blockade of calcium channels would result in the disappearance of calcium activated potassium currents, which would increase the amplitude of the DAP.

In addition, there are literature results, which point out that DAPs in other neurons have turned out to be calciumdependent (Friedmana et al., 1992; Mayer, 1984; Morita \& Barret, 1989; Caeser et al., 2006; Ghamari-Langroudi \& Bourque, 1998; Teruyama \& Armstrong, 2007). Some of these results describe especially sodium and calcium dependent DAPs (Friedmana et al., 1992) (as we also assume), and results, 
which describe calcium dependent DAPs in neuroendocrine cells (Ghamari-Langroudi \& Bourque, 1998; Teruyama \& Armstrong, 2007) can also be found.

Altogether the results described in the above articles support the hypothesis, that calcium dependent mechanisms can stand behind the DAP.

In this paper we will assume that the regulation of $\mathrm{Na}^{+}$ channels takes place trough $\mathrm{Ca}^{2+}$ dependent mechanisms. As it is described in (Cantrell \& Catterall, 2001), the $\alpha$ subunits of sodium channels are exceptionally good substrates for phosphorylation by cAMP-dependent protein kinase (PKA). It can be assumed that through $\mathrm{Ca}^{2+}$ sensitive cyclases, this process is $\mathrm{Ca}^{2+}$ dependent. In contrast, the activation of the $\mathrm{Ca}^{2+}$ dependent phosphatase calcineurin causes dephosphorylation of the channels (Cantrell \& Catterall, 2001). Furthermore in GT-1 cells, cyclic nucleotide gated non-selective cation channels have been described (Vitalis et al., 2000; Charles et al., 2001), which may also be related to $\mathrm{Ca}^{2+}$ dependent currents.

According to these mechanisms, we will assume a sodium current, the activation and inactivation of which are both $\mathrm{Ca}^{2+}$ dependent. Such a current would explain DAPs, and would contribute to bursting behavior. In addition, we will assume that the time constants of the activation and inactivation processes are different, and we will describe these processes with a formalism similar to Hodgkin-Huxley equations. The equation reads

$I_{D A P}=g_{D A P} m_{D A P} h_{D A P}\left(v-E_{N a}\right)$

where $g_{D A P}[\mathrm{nS}]$ is the maximal conductance, $m_{D A P}$ and $h_{D A P}$ are the activation/inactivation variables of the current, and $E_{N a}$ is the reversal potential of sodium, which is assumed to be constant. The activation and inactivation variable is described by

$$
\begin{aligned}
& \dot{m}_{D A P}=\frac{m_{D A P}^{\infty}-m_{D A P}}{\tau_{D A P}^{m}} \quad \dot{h}_{D A P}=\frac{h_{D A P}^{\infty}-h_{D A P}}{\tau_{D A P}^{h}} \\
& m_{D A P}^{\infty}=\frac{c^{n_{D A P} m}}{c^{n_{D A P} m}+K_{D A P}^{n_{D A P} m}} \\
& h_{D A P}^{\infty}=A_{D A P} h \exp \left(-c / s_{D A P_{h}}\right)
\end{aligned}
$$

$\tau_{D A P}^{m}$ and $\tau_{D A P}^{h}$ are assumed to be constant.

\subsection{Model structure}

We can summarize the connections of the various sub-models, as depicted in Fig 3.

It has to be noted that the modular structure ensures the benefit, that certain modules (sub-models) may be substituted by different or more detailed elements in the future.

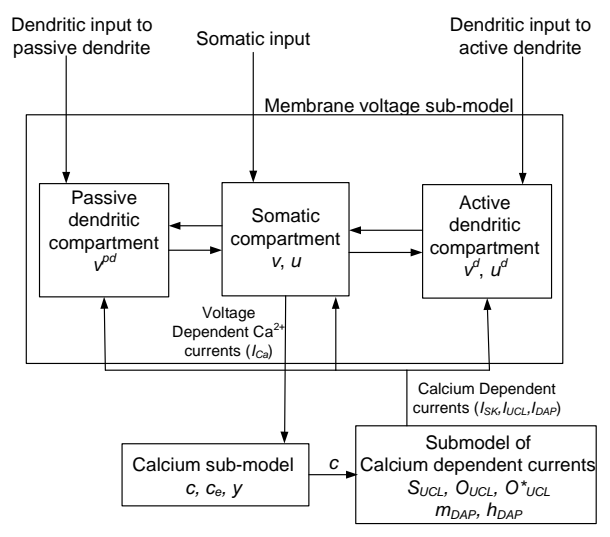

Fig. 3 The modular structure of the model, with the state variables, and the currents connecting their dynamics.

\section{Results and Discussion}

4.1 Membrane voltage sub-model and somato-dendritic interactions

The firing patterns measured in whole cell configuration served as a basis for the determination of the parameters of the membrane voltage sub-model. Furthermore, the results published in (Roberts, Campbell, et al., 2008) were taken into account, mainly corresponding to somato-dendritic and dendrosomatic interplay.

The measured firing pattern of a representative cell (cell 1), and the simulated APs of the model in response to $30 \mathrm{pA}$ can be seen in Fig. 4.

According to the simplicity of the voltage submodel, which describes AP generation and hyperpolarization, one can not expect the model to perfectly describe the shape of APs, and interspike patterns. However the width of APs (approximately $1.5 \mathrm{~ms}$ at $-30 \mathrm{mV}$ ), which is acceptably matched, is critical from the point of view of voltage dependent calcium currents (see the section 3.2), which are fundamental in the determination of intracellular $\mathrm{Ca}^{2+}$ dynamics. Regarding the excitability properties, as it can be seen in Fig. 4, the model produces 4 APs in response to a $200 \mathrm{~ms}$ long $30 \mathrm{pA}$ current step, which is not far from the average AP number observed in experimental data (2.8) corresponding to this current injection protocol. Furthermore the characteristic sub-baseline hyperpolarization is also reproduced.

As it is described in (Roberts, Campbell, et al., 2008), GnRH neurons are capable of dendritic AP generation (in fact, during the measurements described in this article all endogenous APs were detected first at the dendritic site), and somatic and dendritic APs initiate each other in a bilateral way.

In Fig. 4 simulation results can be seen that demonstrate the somato-dendritic interactions. First, as depicted in Fig. $4 \mathrm{~b}$, a dendritic AP is generated with a $5 \mathrm{~ms} 45 \mathrm{pA}$ pulse of 
dendritic injected current, which initiates a somatic AP. Second $(4 \mathrm{c})$, a protocol similar to the one described in (Roberts, Campbell, et al., 2008) (see Fig. 2) is used to evoke somatic APs, which initiate dendritic firing. According to the model simulations, the both the latency from the active dendritic to the somatic compartment is about $1 \mathrm{~ms}$, while the average latency from the soma to the dendrite is about $0.8 \mathrm{~ms}$, which values are in good agreement with the results regarding the time range of these values $(0.37 \mathrm{~ms}$ and $0.45 \mathrm{~ms})$ in the case of $250 \mu \mathrm{m}$ distance between the soma and the dendrite in (Roberts, Campbell, et al., 2008).

\subsubsection{Voltage dependent calcium current}

As mentioned in section 3.2, the voltage dependent $\mathrm{Ca}^{2+}$ current acts as a critical input to the submodel describing $\mathrm{Ca}^{2+}$ dynamics. The determination of the parameters of this current was based on literature data to obtain realistic dynamical properties of this current.

The parameters of the Hodgkin-Huxley channel describing the voltage gated $\mathrm{Ca}^{2+}$ currents were determined to qualitatively match the voltage clamp results published in (Kato et al., 2003). The parameters of the model were adjusted to approximate the voltage dependence of the peak current corresponding to the results of the voltage step protocol described in (Kato et al., 2003). The peak currents of model simulation are compared to experimental data (Kato et al., 2003) in Fig. 5a. According to the simplifying assumption of one channel, the match can be considered acceptable.

\subsection{Calcium dependent potassium currents and AHP}

The parameters of the calcium dynamics and the calcium dependent potassium currents were determined to approximately reproduce the results corresponding to these currents in (Lee et al., 2010), based mainly on the model published in this article. To analyze these currents, the same voltage clamp protocol was used on the model as described in the above article: from $-60 \mathrm{mV}$ holding potential a single voltage step to $40 \mathrm{mV}$ was applied for $600 \mathrm{~ms}$. The results are depicted in Fig 5c. Comparing Fig 5 to Fig. $5 \mathrm{a}$ and $\mathrm{c}$ in (Lee et al., 2010), it can be said that the time courses of these currents show good agreement with experimental data.

To analyze the afterhyperpolarization due to calcium dependent potassium currents, similar to the protocol described in (Lee et al., 2010), four action potentials were evoked by somatic current injections ( $3 \mathrm{~ms}$ wide pulses of $200 \mathrm{pA}$, and $40 \mathrm{~ms}$ between pulses), as depicted in Fig 6. This simulation of the current clamp experiment was performed with normal parameters, and then with the blocking of $I_{S K}$ and $I_{U C L}$ which correspond to the effect of apamin and UCL2077 respectively. It can be said that the qualitative effect of the
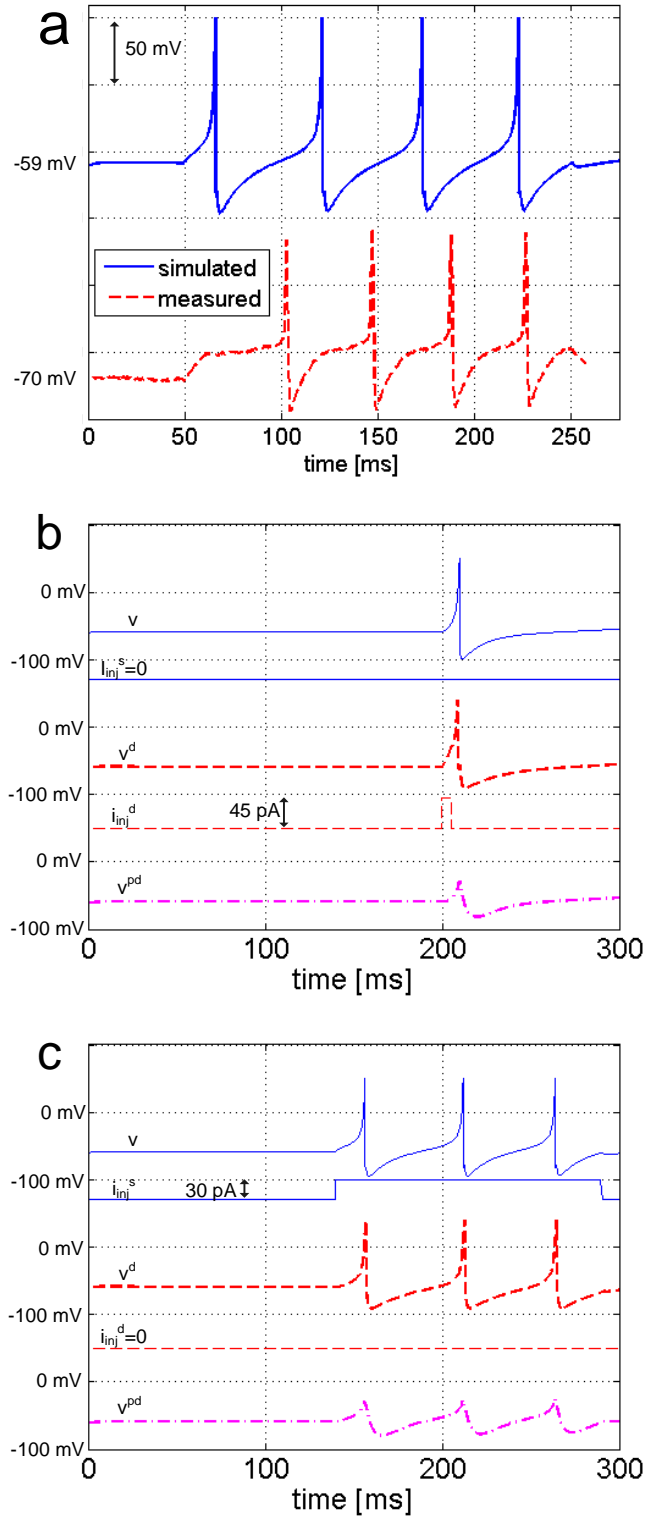

Fig. 4 a) Firing pattern of the model (somatic membrane potential) and of a representative cell (firing frequency, depolarization and hyperpolarization amplitudes close to average) recorded in whole cell configuration, in response to $30 \mathrm{pA}$ somatic current injection. b) Simulation results of dendritic AP generation in the active dendrite and propagation to the soma: Top: somatic membrane potential $(v)$ and injected current $\left(i_{i n j}^{s}\right)$, down: membrane potential and injected current of the active dendritic compartment ( $v^{d}$ and $i_{i n j}^{d}$ ), and membrane potential of the passive dendritic compartment $\left(v^{p d}\right)$. c) Simulation results of somatic AP generation and propagation to dendrite - notations as above. b) c) APs generated in the dendrite via dendritic current injection initiate somatic APs, and vice versa.

hyperpolarizing currents is in good agreement with the experimental results described in (Lee et al., 2010). As it can be seen in Fig 6a, $I_{S K}$ has significant effect on the depolarization in the early stages, while $I_{U C L}$ has a more sustained 

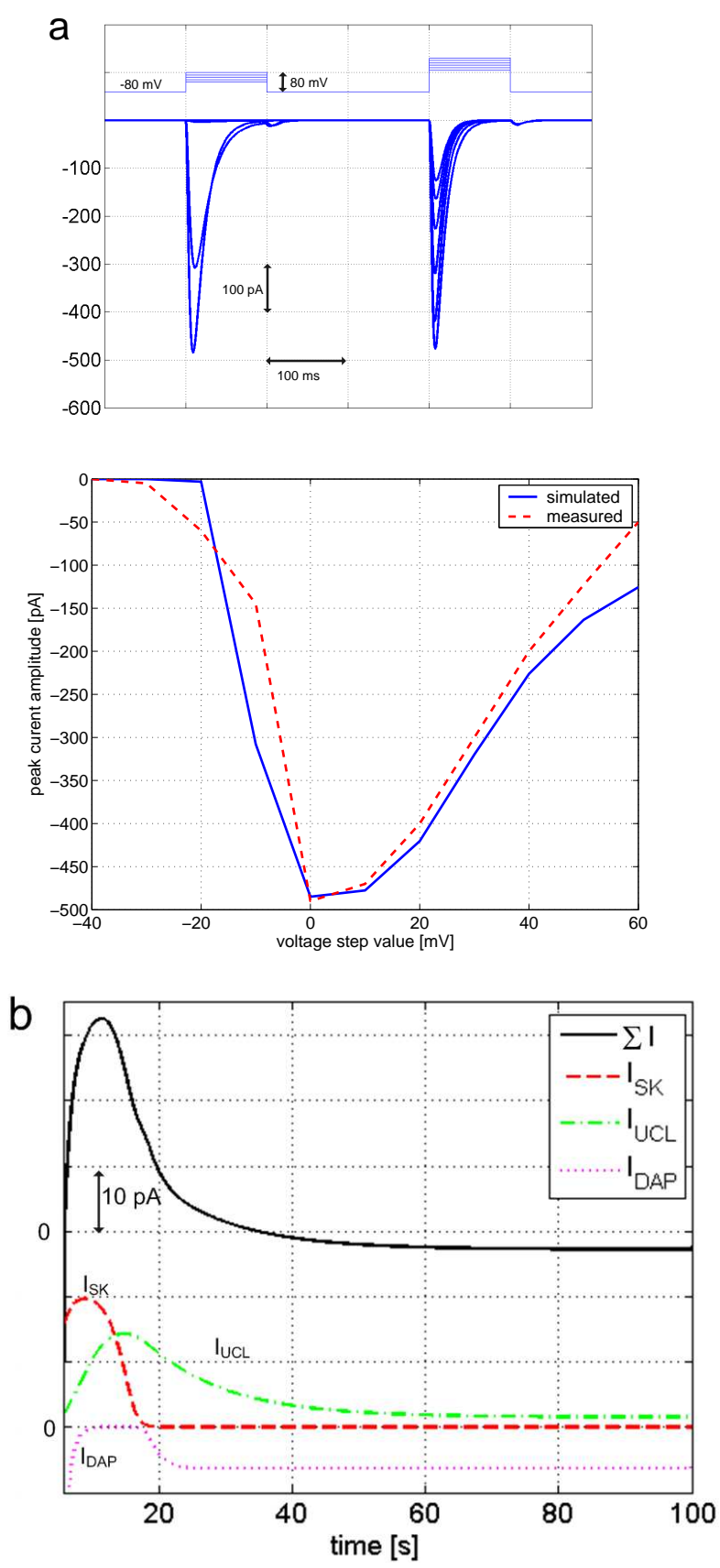

Fig. 5 a) Simulated voltage clamp traces and peak values of voltage gated $\mathrm{Ca}^{2+}$ currents. Peak values are depicted as function of step potential. Experimental data and model simulation. b) Simulated traces of $\mathrm{Ca}^{2+}$ dependent currents after $600 \mathrm{~ms}$ depolarizing voltage step.

effect. According to model simulations, $I_{U C L}$ showed a significant level of activity at rest, and its blockade also raises the resting potential (from about $-61 \mathrm{mV}$ to approximately $-58 \mathrm{mV})$.

In addition, it has to be noted that in the case of these simulations of the afterhyperpolarization phenomena (corresponding to Fig 6a) the $\mathrm{Ca}^{2+}$ dependent depolarizing current
$I_{D A P}$ has been also blocked. The reason for this modification was that without the regulating effect of hyperpolarizing currents, $I_{D A P}$ was shown to be able to evoke additional APs. These APs would modify the calcium dynamics and so via the modifications of hyperpolarizing currents, this results in incomparable voltage traces.

\subsection{Calcium dependent sodium currents and DAP}

To study the DAP caused by the calcium dependent sodium current of the model, the protocol described in (Chu \& Moenter, 2006) was used: 1-4 APs were evoked via somatic current injection pulses, and the DAP was analyzed. Apart from the depolarizing current pulses, which initiate the APs, a constant hyperpolarizing current was used to keep the membrane potential around $-70 \mathrm{mV}$ to render the results comparable to (Chu \& Moenter, 2006). Fig. 6 depicts the DAPs, the underlying changes in cytosolic $\mathrm{Ca}^{2+}$ concentration and $I_{D A P}$ corresponding to various numbers of the preceding APs.

As we can see, the amplitude of the DAP increases with the number of the preceding APs, as described in (Chu \& Moenter, 2006). The quantitative value of the DAP amplitudes in the case of 1,2 and 4 APs $(1.54,2.56$ and $3.05 \mathrm{mV}$ respectively) shows also good agreement with experimental data published in (Chu \& Moenter, 2006).

Furthermore, the latency between the last AP and the peak amplitude (about $200 \mathrm{~ms}$ ) also matches the results described in (Chu \& Moenter, 2006).

\subsection{Effect of calcium dependent currents on short term firing pattern}

To further analyze the effect of the $\mathrm{Ca}^{2+}$ dependent currents on the firing pattern, an additional protocol described in (Lee et al., 2010) was applied to the model. Repetitive spiking was evoked by a $2 \mathrm{~s}$ long current step, and the effect of blocking the apamin-sensitive $\left(I_{S K}\right)$, UCL-sensitive $\left(I_{U C L}\right)$, and $I_{D A P}$ current to the number of spikes was analyzed.

As the second and the third traces of Fig. 7 show, blocking $I_{S K}$ and $I_{U C L}$ increased the number of APs, which is in good agreement with the results described in (Lee et al., 2010). The model also predicts, that the current $I_{D A P}$ strongly contributes to repetitive firing, and blocking it reduces the number of APs. However, if we suppose that this current can be related to the regulation of sodium channels via phosphorylation mechanisms, the selective blockade could be a difficult pharmacological task (if we do not want to block all sodium currents with TTX, to sustain the cell's excitability). 
a
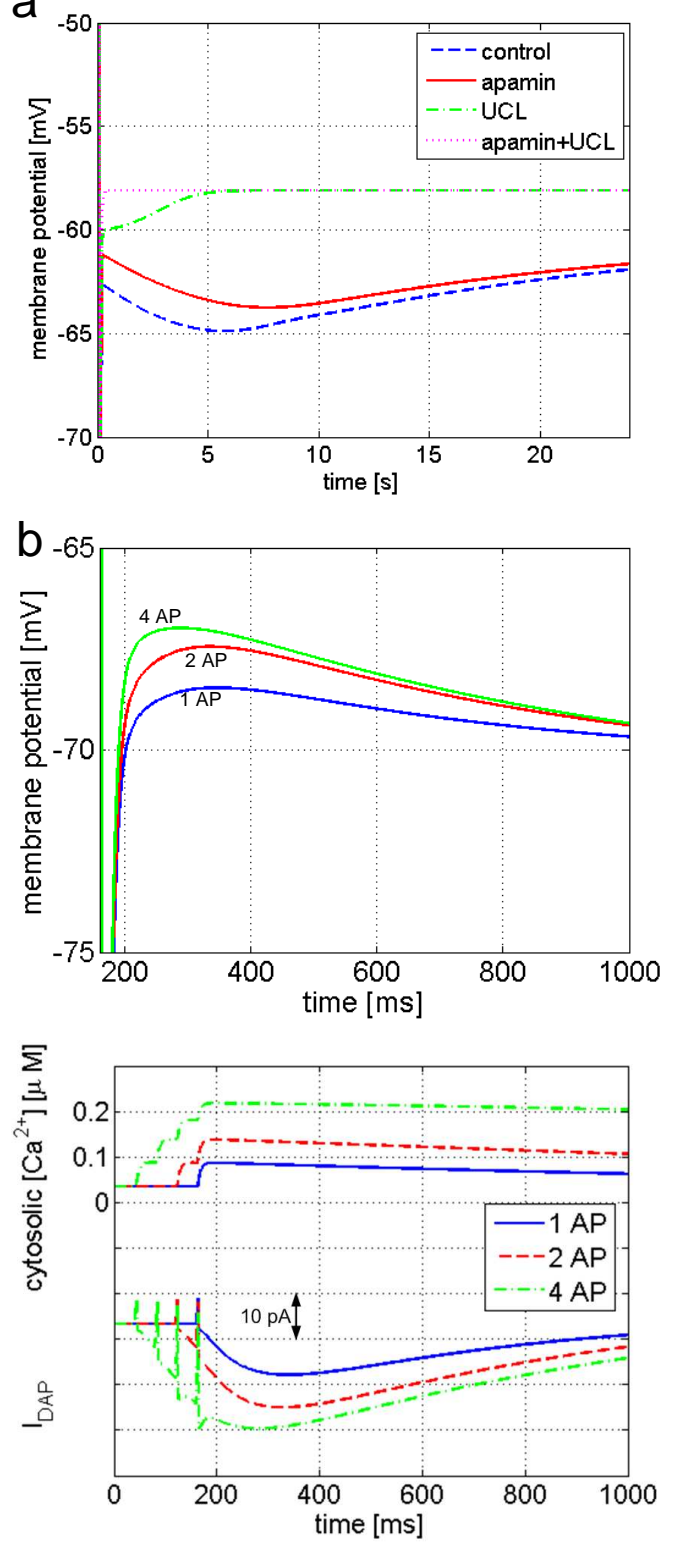

Fig. 6 a) Simulated voltage traces during AHP when blocking various $\mathrm{Ca}^{2+}$ dependent hyperpolarizing currents. b) Simulated DAPs, the underlying $\mathrm{Ca}^{2+}$ transients and the depolarizing $\mathrm{Ca}^{2+}$ dependent sodium current $I_{D A P}$.

\subsection{Long term simulation results and bursting}

As described in 2.3.2, the postsynaptic currents measured in the described configuration correspond to GABA dependent transmission. However, as described in (Kuehl-Kovarik et al., 2002; Suter, 2004), GnRH neurons receive important inputs via AMPA and NMDA type receptors. We will assume that the input to the model is composed of GABA and glutamate derived currents for all compartments:

$$
I_{\text {inj }}^{c o m p}=I_{G A B A}^{c o m p}+I_{G L U}^{c o m p}, \quad c o m p \in\{s, d, p d\}
$$

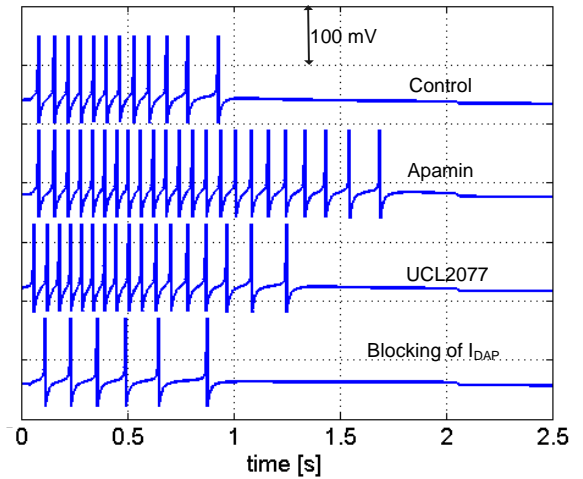

Fig. 7 Simulated firing patterns in the case of blocking various $\mathrm{Ca}^{2+}$ dependent currents.

The measured GABA PSC traces were considered as overall GABA dependent contribution, and in the following we assume that GABA currents are distributed equally among somatic, dendritic and passive dendritic compartments. This will not be true for glutamate (GLU) currents, for which independent artificial traces were generated separately for the three compartments (see later in 4.5.2).

\subsubsection{Intrinsic bursting}

First, we show that the model is capable of intrinsic bursting. If we apply no GABAergic and glutamatergic synaptic input to the model, the firing pattern shows organized bursts of 10-15 APs, separated by 50-70 s long interburst intervals (IBIs), as depicted in Fig. 8. The average IBI value of model simulations is in good agreement with the results described in (Farkas et al., 2010), regarding the simultaneous application of kynurenic acid (Kyn) and bicuculline (Bic), the blockers of ionotropic glutamate receptors, and GABAa receptors respectively.

The correlation between bursts, and $\mathrm{Ca}^{2+}$ transients can be seen clearly in Fig. 8. Furthermore, Fig. 8 shows that while $I_{S K}$, which, according to the modelling assumptions, exhibits an explicit algebraic dependence of the cytosolic calcium level, plays role in the control and termination of bursting. $I_{U C L}$ in contrast, which is described by its own state variables and dynamics, and thus a slower onset and decay, is triggered by the calcium transient, and causes attenuating hyperpolarization between the bursts. $I_{D A P}$ plays a significant role in the initation and maintaining of the bursts.

\subsubsection{Bursting due to realistic synaptic inputs}

To analyze the realistic long term behavior of the model and the manner in which synaptic inputs modulate bursting, some of the measured GABA postsynaptic current traces de- 
picted in Fig. 1 were applied to the model (distributed evenly among compartments).

In order to describe glutamate mediated currents, for which no measurement traces were available, artificial glutamate current traces were generated for each compartment, according to (Suter, 2004). The glutamate current was incorporated into the model via the basic synaptic equation: $I_{G L U}=$ $g_{G L U}\left(v^{c}-E_{G L U}\right)$, where $g_{G L U}$ is the time dependent synaptic conductance, $v^{c}$ is the membrane potential of the actual compartment, and $E_{G L U}$ is the reversal potential for the current (set to $0 \mathrm{mv}$ ). According to (Suter, 2004), we also assumed that in GnRH neurons AMPA-type appears to be the dominant form of glutamatergic receptors, and we generated AMPA-like (Spergel et al., 1999) pulses of glutamate conductance, described by $g_{G L U}(t)=g_{\max } /\left(\tau_{2} / \tau_{1}\right)\left[e^{-t / \tau_{2}}-\right.$ $\left.e^{-t / \tau_{2}}\right]$, where $\tau_{1}=0.8 \mathrm{~ms}$ and $\tau_{2}=2 \mathrm{~ms}$ (see (Suter, 2004)). $g_{\max }$ was chosen from a probability distribution to approximate the glutamatergic PSC amplitudes described in (Suter, 2004). The time distribution of glutamatergic pulses was also derived by probabilistic means, to reproduce the average pulse frequency described in (Suter, 2004) (about 0.35 pulse/sec).

Fig. 9a depicts one representative simulation result (response to the first 80 s of the third trace of Fig. 1b. At about 6 , 30,44 and $60 \mathrm{~s}$, typical bursts can be seen, which are accompanied by distinct calcium transients. The bursts are initiated and maintained by the positive feedback mechanism of the calcium dependent DAP, further APs, and further calcium inflow, causing further depolarization, as long as $I_{D A P}$ is not inactivated. The bursts in this simulation are dominantly initiated by GABA currents.

The calcium transient, which is enhanced by the IP3 gated $\mathrm{Ca}^{2+}$ currents later results in AHP (due to $I_{S K}$ and
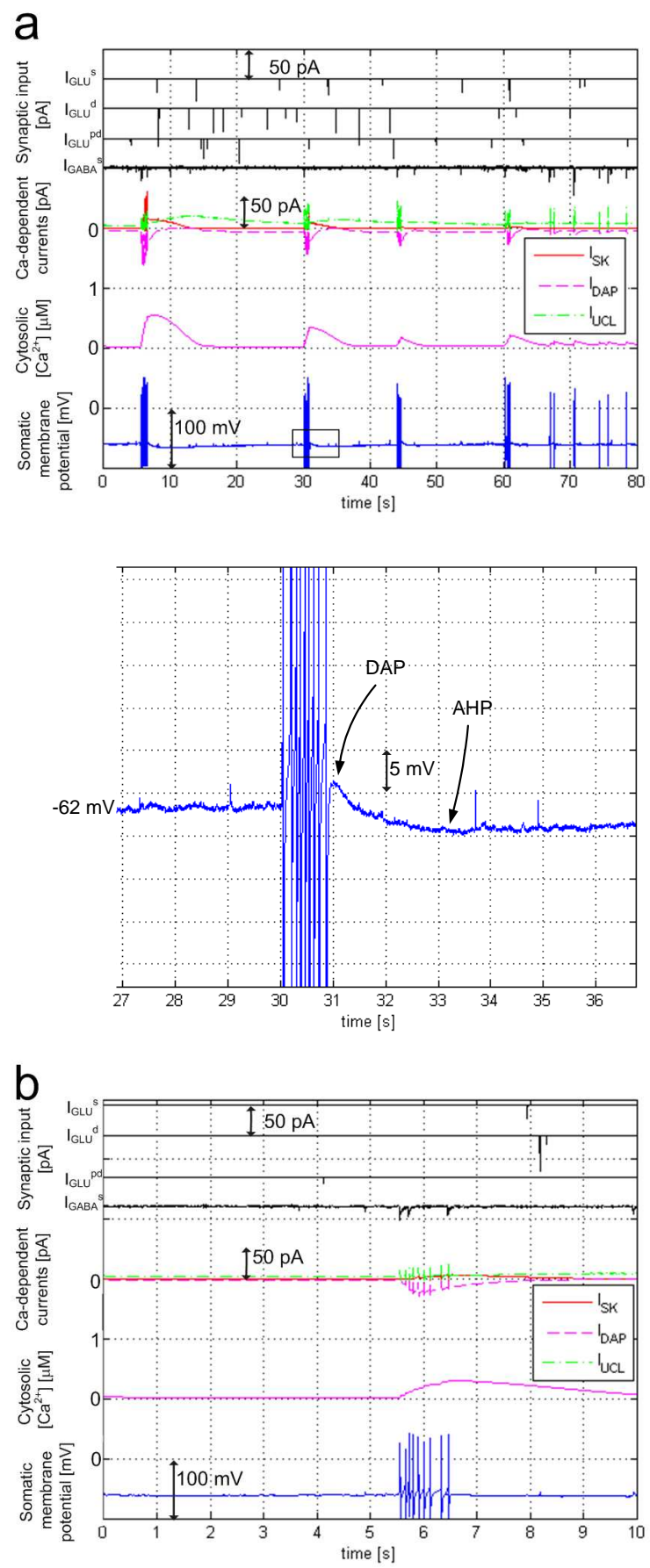

Fig. 9 a) Long term model behavior in the case of realistic somatodendritic inputs. The second figure depicts the enlarged version of the rectangle to visualize DAP and AHP. b) The first burst in the case of $I_{D A P}$ decreased by 50\%: The number of APs in the burst is reduced, intraburst frequency and the calcium transient are attenuated.

$\left.I_{U C L}\right)$, which terminates firing and hyperpolarizes the membrane, causing the further inputs implying only subthreshold responses. Due to the dynamics of $I_{U C L}$ the (negative) peak of the hyperpolarization follows the calcium transient with a delay. 
As the hyperpolarization is attenuated, the cell becomes excitable again, and the GABA inputs initiate a second burst at $30 \mathrm{~s}$ (as we will show later glutamate inputs are also capable of initiating bursting).

After the fourth burst and the following hyperpolarization in Fig. 9a, in response to increased inputs, more scattered APs can be seen, which are unable to generate large intracellular calcium transients, typical bursts and hyperpolarization.

In general it can be said that loose patch recordings corresponding to GnRH neurons showing typical bursting behavior lack (or show only very few) single APs. In such recordings (in (Lee et al., 2010) or in our own measurements) APs dominantly appear in well distinguished bursts, in which the maximum delay between two APs is about $1 \mathrm{~s}$. However the assumption that a single AP always initiates a burst, which would be suggested by the loose-patch recordings is in contradiction with the whole cell results regarding the cited AHP and DAP measurement results, on which important model parameters are based. That means single APs appearing in the model simulation are acceptable output of the model, however model parameters based on whole cell recordings may be reconsidered in the future. It can be assumed that whole cell patch clamp recordings severely influence the cell's internal medium, and so calcium dynamics, calcium dependent currents and excitability. Altogether, it can be said that in the resulting firing pattern in response to realistic input, the elements of periodic bursting behavior can be unambiguously identified.

The simulation results show that $I_{D A P}$ has a critical role in initiating and maintaining the burst as well as in the mediation of intraburst frequency, as depicted in Fig. 9. As $I_{D A P}$ is decreased by $50 \%$, the number of APs within bursts, as well as intraburst frequency decreases (as suggested by Fig. 7). This results in more realistic AP/burst number and intraburst frequency (compared to the values described in 2.4), but in general also raises the possibility of the appearance of single spikes. In this case further synaptic inputs are needed during the time of DAP to generate more APs in the burst. Finally, as depicted in Fig. 9b, through the attenuation of the $\mathrm{Ca}^{2+}$ transient (fewer spikes $\rightarrow$ lower $\mathrm{Ca}^{2+}$ inflow), $I_{D A P}$ also mediates the amplitude and duration of the AHP.

Figure 10 illustrates that in the case of low GABA activity (the first trace of Fig. 1b is applied), glutamate inputs are also capable of initiating a burst. This figure also illustrates that for the maintenance of the burst, intensive clustered synaptic inputs are not always necessary, the intrinsic properties may ensure repetitive firing via calcium transients and DAP.

According to the overviewed literature results, which are in major part confirmed by model simulations it can be assumed that the complex interplay of currents responsible for DAP and AHP together with the synaptic inputs determine

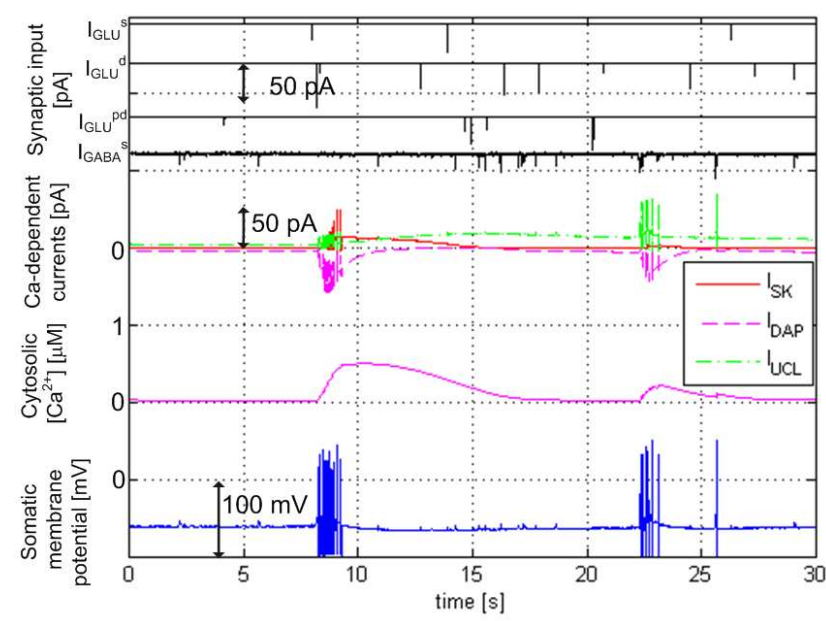

Fig. 10 Demonstration of burst initiation by dendritic glutamate input: The first burst is initiated by a dendritic glutamate spike during low GABA activity. the second burst is triggered by GABA inputs.

the firing pattern of GnRH neurons. The provided model framework (after possible further tuning of parameters) seems to be appropriate for analyzing how a certain change in a particular current may influence the resulting pattern.

Regarding model simulations, it can be stated that multiple factors determine whether an AP is able to trigger a large calcium transient or not. The resulting synaptical input and the somato-dendritic interplay affects the exact shape of the AP, which determines the inflow of calcium via voltage dependent $\mathrm{Ca}^{2+}$ currents. Furthermore, appearance of a large, IP3 amplified $\mathrm{Ca}^{2+}$ transient also depends on the internal state variable $y$ of the $\mathrm{Ca}^{2+}$ subsystem (the ratio of free $\mathrm{Ca}^{2+}$-binding inactivation domains), and on the actual cytosolic and $\mathrm{ER} \mathrm{Ca}^{2+}$ concentrations.

\subsection{Background of $I_{D A P}$}

As we have shown in the previous subsection, the submodel describing the $\mathrm{Ca}^{2+}$ dynamics combined with the $\mathrm{Ca}^{2+}$ dependent currents and the simplified membrane voltage submodel is able to describe several experimental results corresponding to DAP and AHP. It can be said that while $I_{D A P}$, which underlies the DAP, in the current model is basically assumed to correspond to the $\mathrm{Ca}^{2+} \uparrow \rightsquigarrow \mathrm{Ca}^{2+}$-dependent cyclase $\rightsquigarrow$ cAMP $\rightsquigarrow$ PKA $\rightsquigarrow g_{N a} \downarrow$ and the $\mathrm{Ca}^{2+} \uparrow \rightsquigarrow$ calcineurin $\rightsquigarrow g_{N a} \uparrow$ pathways, but in general, the describing mathematical formalism may also be interpreted as corresponding to any other $\mathrm{Ca}^{2+}$-dependent mechanism, which influences the TTX dependent sodium conductances. The only critical property of this current in the model is the $\mathrm{Ca}^{2+}$ dependence, which is confirmed by $\mathrm{Cd}^{2+}$ related measurements in (Chu \& Moenter, 2006). 


\subsection{Significance of the proposed model}

First of all, it has to be noted that the model described in this article is entirely based on measurement results originating from hypothalamic slices, in contrast to several previous models (LeBeau et al., 2000; Van Goor et al., 2000; Fletcher $\& \mathrm{Li}, 2009)$. The main aim of the model development procedure was to reproduce the characteristic features of the bursting behavior shown by GnRH neurons in loose patch recordings. This aim was fulfilled with the application of a simplified model for the description of AP generation, which reduced the model complexity compared to previous results (Lee et al., 2010; Duan et al., 2011) regarding the models of GnRH neuronal bursting. In addition, the proposed model is capable of the description of depolarizing afterpotentials, simple somato-dendritic interactions (see (Roberts et al., 2006; Roberts, Hemond, \& Suter, 2008; Roberts et al., 2009)), and approximated reproduction of the shape of single APs. Furthermore, during model development, the parameters of the voltage dependent calcium current were determined according to (Kato et al., 2003). Lastly, for the simulation of the model, real recorded GABA PSC traces were used to reproduce realistic inputs.

\subsection{Additional considerations}

In addition to the assumed $\mathrm{Ca}^{2+}$ dependent $I_{D A P}$, the $\mathrm{Ca}^{2+}$ dependence of AHP is also critical in the determination of the firing pattern. This points out that $\mathrm{Ca}^{2+}$, and $\mathrm{Ca}^{2+} \mathrm{de}-$ pendent currents are fundamental central regulatory elements in GnRH electrophysiology. $\mathrm{Ca}^{2+}$ dynamics on the other hand are influenced in GnRH neurons by various endocrine ligands and mechanisms. Regarding the autocrine mechanisms, GnRH affects $\mathrm{Ca}^{2+}$ levels through cAMP (Fletcher $\& \mathrm{Li}, 2009)$, while estrogens can influence GnRH neuronal functions via direct (Chu et al., 2009) and indirect mechanisms (Petersen et al., 2003; Wintermantel et al., 2006; Heldring et al., 2007), both of which can involve altered $\mathrm{Ca}^{2+}$ signaling, in which CREB and PKA may play a central role (Ábrahám et al., 2003).

Kisspeptins, which play a critically important role in indirect estrogen signaling mechanisms, stimulate GnRH neurons via a G-protein couple receptor (GPR54), which involves the activation of phospholipase-C, with a subsequent mobilization of intracellular $\mathrm{Ca}^{2+}$. Kisspeptin neurons are not only implicated heavily in puberty onset (de Roux et al., 2003; Seminara et al., 2003; Semple et al., 2005), but also play crucial roles in mediating positive (Herbison, 2008) and negative (Rance et al., 2010) estrogen feedback signals to the GnRH neuronal system. Furthermore, kisspeptin neurons in the ARC also seem to represent the long-thoughtafter pulse generator that governs the episodic secretion of GnRH (Lehman et al., 2010; Maeda et al., 2010).
A better characterization of estrogen effects on these $\mathrm{Ca}^{2+}$ signaling pathways and their effect on electrophysiological properties will contribute to a better understanding of molecular mechanisms whereby cyclic changes in ovarian hormones regulate the secretory activity of $\mathrm{GnRH}$ neurons during the estrous cycle.

\section{Conclusions}

\subsection{Conclusions}

In this paper, a modular model of GnRH neuronal electrophysiology is described. The voltage sub-model is described by an impulsive system, while the calcium sub-model is described by a system of normal ODEs. Both sub-models are excitable, which means the action potential in the case of the voltage sub-model, and the calcium transient in the case of the calcium sub-model. The two systems are coupled to each other, via voltage dependent calcium fluxes and calcium dependent currents. Compared to experimental results the model is able to reproduce both the DAP and AHP phenomena, which are thought to underlie the regulation of bursting.

Applying realistic input to the model, originating from the recording of post-synaptic currents, the resulting dynamics gives rise to a firing pattern in which the elements of periodic bursting behavior can be unambiguously identified.

\section{References}

Bainov, D., \& Simeonov, P. (Eds.). (1989). Systems with impulse effect. Ellis Horwood Limited.

Bezprozvanny, I., Watras, J., \& Ehrlich, B. (1991). Bellshaped calcium-response curves of Ins(1,4,5)-P3-and calcium gated channels from endoplasmic reticulum of cerebellum. Nature, 351, 751-754.

Caeser, M., Brown, D., G ahwiler, B., \& Kn opfel, T. (2006). Characterization of a calcium-dependent current generating a slow afterdepolarization of CA3 pyramidal cells in rat hippocampal slice cultures. European Journal of Neuroscience, 5, 560-569.

Campbell, R., Gaidamaka, G., Han, S., \& Herbison, A. (2009). Dendro-denritic bundling and shared synapses between gonadotropin-releasing hormone neurons. Proceedings of the National Academy of Sciences of the USA, 106, 10835-10840.

Campbell, R., Han, S., \& Herbison, A. (2005). Biocytin filling of adult gonadotropin-releasing hormone neurons in situ reveals extensive, spiny, dendritic processes. Endocrinology, 146, 1163-1169. 
Cantrell, A., \& Catterall, W. (2001). Neuromodulation of $\mathrm{Na}^{+}$channels: An unexpected form of cellular plasticity. Nature Reviews Neuroscience, 2, 397-407.

Charles, A., Weiner, R., \& Costantin, J. (2001). Camp modulates the excitability of immortalized hypothalamic (GT1) neurons via a cyclic nucleotide gated channel. Molecular Endocrinology, 15, 997-1009.

Chu, Z., Andrade, J., Shupnik, M. A., \& Moenter, S. M. (2009). Differential regulation of gonadotropinreleasing hormone neuron activity and membrane properties by acutely applied estradiol: dependence on dose and estrogen receptor subtype. J Neurosci, 29(17), 5616-27.

Chu, Z., \& Moenter, S. (2006). Physiologic regulation of a tetrodotoxin-sensitive sodium influx that mediates a slow afterdepolarization potential in gonadotropinreleasing hormone neurons: possible implications for the central regulation of fertility. Journal of Neuroscience, 26, 11961-73.

Conn, P., \& Freeman, M. (2000). Neuroendocrinology in physiology and medicine. 999 Riverview Drive Suite 208 Totowa New Jersey 07512: Humana Press.

Constantin, J., \& Charles, A. (1999). Spontaneous action potentials initiate rhythmic intercellular calcium waves in immortalized hypothalamic (GT1-1) neurons. Journal of Neurophysiology, 82, 429-435.

Csercsik, D., Farkas, I., Szederkényi, G., Hrabovszky, E., Liposits, Z., \& Hangos, K. (2010). Hodgkin-Huxley type modelling and parameter estimation of $\mathrm{GnRH}$ neurons. BioSystems, 100, 198-207.

de Roux, N., Genin, E., Carel, J., Matsuda, F., Chaussain, J., \& Milgrom, E. (2003). Hypogonadotropic hypogonadism due to loss of function of the KiSS1-derived peptide receptor GPR54. Proc Natl Acad Sci U S A, 100(19), 10972-6.

DeFazio, R., Heger, S., Ojeda, S., \& Moenter, S. (2002). Activation of A-type $\gamma$-aminobutyric acid receptors excites gonadotropin-releasing hormone neurons. Molecular Endocrinology, 16, 2872-2891.

DeFazio, R., \& Moenter, S. (2002). Estradiol feedback alters potassium currents and firing properties of gonadotropin- releasing hormone neurons. Molecular Endocrinology, 16, 2255-2265.

Duan, W., Lee, K., Herbison, A., \& Sneyd, J. (2011). A mathematical model of adult $\mathrm{GnRH}$ neurons in mouse brain and its bifurcation analysis. Journal of Theortical Biology, 276, 22-34.

Farkas, I., Kalló, I., Deli, L., Vida, B., Hrabovszky, E., Fekete, C., et al. (2010). Retrograde endocannabinoid signaling reduces GABAergic synaptic transmission to gonadotropin-releasing hormone neurons. Endocrinology, 151, 5818-5829.
Finch, E., Turner, T., \& Goldin, S. (1991). Calcium as a coagonist of inositol 1,4,5-trisphosphate-induced calcium release. Science, 252, 443-446.

Fletcher, P., \& Li, Y. (2009). An integrated model of electrical spiking, bursting, and calcium oscillations in GnRH neurons. Biophysical Journal, 96, 4514-4524.

Friedmana, A., Arens, J., Heinemann, U., \& Gutnick, M. (1992). Slow depolarizing afterpotentials in neocortical neurons are sodium and calcium dependent. $\mathrm{Neu}$ roscience Letters, 135, 13-17.

Ghamari-Langroudi, M., \& Bourque, C. (1998). Caesium blocks depolarizing after-potentials and phasic firing in rat supraoptic neurones. Journal of Physiology, 510, 165-175.

Han, S., Abraham, I., \& Herbison, A. (2002). Effect of GABA on GnRH neurons switches from depolarization to hyperpolarization at puberty in the female mouse. Endocrinology, 143, 1459-1466.

Han, S., Todman, M., \& Herbison, A. (2004). Endogenous GABA release inhibits the firing of adult gonadotropin-releasing hormone neurons. Endocrinology, 145, 495-499.

Heldring, N., Pike, A., Andersson, S., Matthews, J., Cheng, G., Hartman, J., et al. (2007). Estrogen receptors: how do they signal and what are their targets. Physiol Rev, 87(3), 905-31.

Herbison, A. (2008). Estrogen positive feedback to gonadotropin-releasing hormone $(\mathrm{GnRH})$ neurons in the rodent: the case for the rostral periventricular area of the third ventricle (RP3V). Brain Res Rev, 57(2), 277-87.

Herbison, A., Pape, J., Simonian, S., Skynner, M., \& Sim, J. (2001). Molecular and cellular properties of GnRH neurons revealed through transgenics in mouse. Molecular and Cellular Endocrinology, 185, 185-194.

Izhikevich, E. (2005). Dynamical systems in neuroscience. Camebridge, Massachusetts, London, England: The MIT Press.

Jasoni, C., Romano, N., Constantin, S., Lee, K., \& Herbison, A. (2010). Calcium dynamics in gonadotropinreleasing hormone neurons. Frontiers in Neuroendocrinology, 31, 259-269.

Kato, M., Ui-Tei, K., Watanabe, M., \& Sakuma, Y. (2003). Characterization of voltage-gated calcium currents in gonadotropin-releasing hormone neurons tagged with green fluorescent protein in rats. Endocrinology, 144, 5118-5125.

Knobil, E. (1980). The neuroendocrine control of the menstrual cycle. Hormone Research, 36, 53-88.

Krsmanovic, L., Stojilkovic, S., Merelli, F., Dufour, S., Virmani, M., \& Catt, K. (1992). Calcium signaling and episodic secretion of gonadotropin-releasing hormone 
in hypothalamic neurons. Proceedings of the National Academy of Sciences of the USA, 89, 8462-8466.

Kuehl-Kovarik, M., Partin, K., Handa, R., \& Dudek, F. (2005). Spike-dependent depolarizing afterpotentials contribute to endogenous bursting in gonadotropin releasing hormone neurons. Neuroscience, 134, 295300.

Kuehl-Kovarik, M., Pouliot, W., Halterman, G. L., Handa, R., Dudek, F., \& Partin, K. (2002). Episodic bursting activity and response to excitatory amino acids in acutely dissociated gonadotropin-releasing hormone neurons genetically targeted with green fluorescent protein. Journal of Neuroscience, 22, 2313-2322.

LeBeau, A., Goor, F. V., Stojilkovic, S., \& Sherman, A. (2000). Modeling of membrane excitability in gonadotropin-releasing hormone-secreting hypothalamic neurons regulated by $\mathrm{Ca}^{2+}$-mobilizing and adenylyl cyclase-coupled receptors. The Journal of Neuroscience, 20, 9290-9297.

Lee, K., Duan, W., Sneyd, J., \& Herbison, A. (2010). Two slow calcium-activated afterhyperpolarization currents control burst firing dynamics in gonadotropinreleasing hormone neurons. Journal of Neuroscience, 30, 6214-6224.

Lehman, M., Coolen, L., \& Goodman, R. (2010). Minireview: Kisspeptin/neurokinin B/dynorphin (KNDy) cells of the arcuate nucleus: A central node in the control of ronadotropin-releasing hormone secretion. Endocrinology, 151, 3479-3489.

Li, Y., \& Rinzel, J. (2010). Equations for InsP receptor mediated $\left[\mathrm{Ca}^{2+}\right]_{i}$-oscillations derived from a detailed kinetic model: a Hodgkin-Huxley like formalism. Journal of Theoretical Biology, 166, 461-473.

Maeda, K., Ohkura, S., Uenoyama, Y., Wakabayashi, Y., Oka, Y., Tsukamura, H., et al. (2010). Neurobiological mechanisms underlying gnrh pulse generation by the hypothalamus. Brain Res.

Mayer, M. (1984). A calcium-activated chloride current generates the after-depolarization of rat sensory neurones in culture. Journal of Physiology, 364, 217239.

Moenter, S., \& DeFazio, R. (2005). Endogenous $\gamma$ aminobutyric acid can excite gonadotropin-releasing hormone neurons. Endocrinology, 146, 5374-5379.

Morita, K., \& Barret, E. (1989). Calcium dependent depolarizations originating in lizard motor nerve terminals. , 9, 3359-3369.

Navarro, V., Gottsch, M., Chavkin, C., Okamura, H., Clifton, D., \& Steiner, R. (2009). Regulation of gonadotropin-releasing hormone secretion by Kisspeptin/Dynorphin/Neurokinin B neurons in the arcuate nucleus of the mouse. The Journal of Neuroscience, 29, 11859-11866.
Nunemaker, C., DeFazio, R., \& Moenter, S. (2003). Calcium current subtypes in GnRH neurons. Biology of Reproduction, 69, 1914-1922.

Parker, I., \& Ivorra, I. (1990). Inhibition by $\left[\mathrm{Ca}^{2+}\right]_{i}$ by inositol trisphosphate-mediated $\left[\mathrm{Ca}^{2+}\right]_{i}$ liberation: a possible mechanism for oscillatory release of $\mathrm{Ca}^{2+}$. Proceedings of the National Academy of Sciences of the USA, 87, 260-264.

Petersen, S., Ottem, E., \& Carpenter, C. (2003, Dec). Direct and indirect regulation of gonadotropin-releasing hormone neurons by estradiol. Biol Reprod, 69(6), 1771-8.

Rance, N., Krajewski, S., Smith, M., Cholanian, M., \& Dacks, P. (2010). Neurokinin B and the hypothalamic regulation of reproduction. Brain Res.

Roberts, C., Best, J., \& Suter, K. (2006). Dendritic processing of excitatory synaptic input in hypothalamic gonadotropin releasing-hormone neurons. Endocrinology, 147, 1545-1555.

Roberts, C., Campbell, R., Herbison, A., \& Suter, K. (2008). Dendritic action potential initiation in hypothalamic gonadotropoin-releasing hormone neurons. Endocrinology, 149, 3355-3360.

Roberts, C., Hemond, P., \& Suter, K. (2008). Synaptic integration in hypothalamic gonadotropin releasing hormone $(\mathrm{GnRH})$ neurons. Neuroscience, 254, 13371351.

Roberts, C., O'Boyle, M., \& Suter, K. (2009). Dendrites determine the contribution of after depolarization potentials (ADPs) to generation of repetitive action potentials in hypothalamic gonadotropin releasing-hormone (GnRH) neurons. Journal of Computational Neuroscience, 26, 39-53.

Seminara, S., Messager, S., Chatzidaki, E., Thresher, R., Acierno, J. J., Shagoury, J., et al. (2003). The GPR54 gene as a regulator of puberty. $N$ Engl J Med, 349(17), 1614-27.

Semple, R., Achermann, J., Ellery, J., Farooqi, I., Karet, F., Stanhope, R., et al. (2005). Two novel missense mutations in $\mathrm{g}$ protein-coupled receptor 54 in a patient with hypogonadotropic hypogonadism. J Clin Endocrinol Metab, 90(3), 1849-55.

Sim, J., Skynner, M., \& Herbison, A. (2001). Heterogeneity in the basic membrane properties of postnatal gonadotropin-releasing hormone neurons in the mouse. The Journal of Neuroscience, 21, 1067-1075.

Spergel, D., Krüth, U., Hanley, D., Sprengel, R., \& Seeburg, P. (1999). Gaba- and glutamate-activated channels in green fluorescent protein-tagged gonadotropinreleasing hormone neurons in transgenic mice. The Journal of Neuroscience, 19, 2037-2050.

Stojilkovic, S., Krsmanovic, L., Spergel, D., \& Catt, K. (1994). GnRH neurons: intrinsic pulsatility and 
receptor-mediated regulation. Trends in Endocrinology and Metabolism, 5, 201-209.

Suter, K. (2004). Control of firing by small (s)- $\gamma$-amino-3hydroxy-5-methylisoxazolepropionic acid-like inputs in hypothalamic gonadotropin releasing-hormone (GnRH) neurons. Neuroscience, 128, 443-450.

Suter, K., Song, W., Sampson, T., Wuarin, J., Saunders, J., Dudek, F., et al. (2000). Genetic targeting of green fluorescent protein to gonadotropin-releasing hormone neurons: characterization of whole-cell electrophysiological properties and morphology. Endocrinology, 141, 412-419.

Teruyama, R., \& Armstrong, W. (2007). Calcium-dependent fast depolarizing afterpotentials in vasopressin neurons in the rat supraoptic nucleus. Journal of Neurophysiology, 98, 2612-2621.

Van Goor, F., LeBeau, A., Krsmanovic, L., Sherman, A., Catt, K., \& Stojilkovic, S. (2000). Amplitudedependent spike-broadening and enhanced $\mathrm{Ca}^{2+}$ signaling in GnRH-secreting neurons. Biophysical Journal, 79, 1310-1323.

Verkhratsky, A. (2005). Physiology and pathophysiology of the calcium store in the endoplasmic reticulum of neurons. Physiological Reviews, 85, 201-279.

Vitalis, E., Costantin, J., Tsai, P., Sakakibara, H., Paruthiyil, S., Iiri, T., et al. (2000). Role of the cAMP signaling pathway in the regulation of gonadotropin-releasing hormone secretion in GT1 cells. Proceedings of the National Academy of Sciences of the United States of America, 97, 1861-1866.

Watanabe, M., Sakuma, Y., \& Kato, M. (2004). High expression of the R-type voltage-gated $\mathrm{Ca}^{2+}$ channel and its involvement in $\mathrm{Ca}^{2+}$-dependent gonadotropinreleasing hormone release in GT1-7 cells. Endocrinology, 145, 2375-2388.

Watanabe, M., Sakuma, Y., \& Kato, M. (2009). GABAA receptors mediate excitation in adult rat $\mathrm{GnRH}$ neurons. Biology of Reproduction, 81, 327-332.

Wintermantel, T., Campbell, R., Porteous, R., Bock, D., Grone, H., Todman, M., et al. (2006). Definition of estrogen receptor pathway critical for estrogen positive feedback to gonadotropin-releasing hormone neurons and fertility. Neuron, 52(2), 271-80.

Yin, C., Ishii, H., Tanaka, N., Sakuma, Y., \& Kato, M. (2008). Activation of A-type $\gamma$-amino butyric acid receptors excites gonadotrophin-releasing hormone neurones isolated from adult rats. Journal of $\mathrm{Neu}$ roendocrinology, 20, 566-575.

Ábrahám, I., Han, S., Todman, M., Korach, K., \& Herbison, A. (2003). Estrogen receptor beta mediates rapid estrogen actions on gonadotropin-releasing hormone neurons in vivo. J Neurosci, 23(13), 5771-7.

\section{Appendix: Model Parameters}

Table 1 Parameters of the membrane voltage submodel

\begin{tabular}{|c|c|c|c|}
\hline parameter & value & parameter & value \\
\hline$C$ & $10 \mathrm{pF}$ & $C^{d}$ & $6 \mathrm{pF}$ \\
$C^{p d}$ & $4 \mathrm{pF}$ & & \\
$v_{r}$ & $-55 \mathrm{mV}$ & $v_{r}^{d}$ & $-55 \mathrm{mV}$ \\
$v_{t}$ & $-50 \mathrm{mV}$ & $v_{t}^{d}$ & $-53 \mathrm{mV}$ \\
$k$ & 0.15 & $k^{d}$ & 0.08 \\
$a$ & $0.15 m s^{-1}$ & $a^{d}$ & $0.1 m s^{-1}$ \\
$b$ & $-0.4 p A m V^{-1}$ & $b^{d}$ & $-0.9 p A m V^{-1}$ \\
$c$ & -80 & $c^{d}$ & -60 \\
$d$ & 500 & $d^{d}$ & 150 \\
$v_{p e a k}$ & $50 \mathrm{mV}$ & $v_{p e a k}^{d}$ & $40 \mathrm{mV}$ \\
$c_{s}$ & $3 p A m V^{-1}$ & $c_{d}$ & $2 p A m V^{-1}$ \\
$c_{p s}$ & $0.3 p A m V^{-1}$ & $c_{p d}$ & $1 p A m V^{-1}$ \\
$r_{d}$ & $1 / 4$ & $r_{p d}$ & $1 / 4$ \\
\hline
\end{tabular}

Table 2 Parameters of the voltage dependent $\mathrm{Ca}^{2+}$ current

\begin{tabular}{|c|c|c|c|}
\hline parameter & value & parameter & value \\
\hline$g_{C a}$ & $11.5 \mathrm{nS}$ & & \\
$V_{1 / 2_{m C a}}$ & $-13 \mathrm{mV}$ & $V_{1 / 2_{h C a}}$ & $-28 \mathrm{mV}$ \\
$K_{m C a} C a$ & 2.6 & $K_{h C a}$ & 5.2 \\
$C_{b a s e}^{m C a}$ & $10.2 \mathrm{~ms}$ & $C_{b a s e}^{h C a}$ & $17 \mathrm{~ms}$ \\
$C_{a m p}^{m C a}$ & $-6.0 \mathrm{~ms}$ & $C_{a m p}^{h C a}$ & $45 \mathrm{~ms}$ \\
$V_{\text {max } m C a}$ & $17 \mathrm{mV}$ & $V_{\max h C a}$ & $-63 \mathrm{mV}$ \\
$\sigma_{m C a}$ & 34 & $\sigma_{h C a}$ & 55 \\
\hline
\end{tabular}

Table 3 Parameters of the calcium submodel

\begin{tabular}{|c|c|}
\hline parameter & value \\
\hline$\rho$ & 0.02 \\
\hline$\gamma$ & 27 \\
\hline$I P 3$ & $0.4 \mu M$ \\
\hline$K_{f}$ & $1.4 \cdot 10^{-4} \mathrm{~ms}^{-1}$ \\
\hline$K_{i}$ & $0.4 \mu M$ \\
\hline$K_{a}$ & $0.35 \mu M$ \\
\hline$J_{e r}$ & $4 \cdot 10^{-7} \mathrm{~ms}^{-1}$ \\
\hline$A$ & $1.5 \cdot 10^{-4} \mu M^{-1} m s^{-1}$ \\
\hline$K_{d}$ & $0.45 \mu M$ \\
\hline$P_{\text {rate }}$ & 1 \\
\hline$a_{1}$ & $0.2 \cdot 10^{-4}$ \\
\hline$a_{2}$ & $35 \mathrm{~ms}$ \\
\hline$a_{3}$ & $600 \mathrm{~ms}$ \\
\hline$a_{4}$ & $4 \mathrm{~ms}$ \\
\hline$a_{5}$ & $35 \mathrm{~ms} \mu M^{-1}$ \\
\hline$V_{P}$ & $2.5 \cdot 10^{-3} \mu M m s^{-1}$ \\
\hline$K_{P}$ & $1.425 \mu M$ \\
\hline$V_{\mathrm{NaCa}}$ & $3.5 \cdot 10^{-4} \mu M m s^{-1}$ \\
\hline$K_{\mathrm{NaCa}}$ & $0.17 \mu M$ \\
\hline$\alpha$ & $5 \cdot 10^{-3} \mu M m s^{-1} p A^{-1}$ \\
\hline
\end{tabular}


Table 4 Parameters of the calcium dependent currents

\begin{tabular}{|c|c|}
\hline parameter & value \\
\hline$g_{S K}$ & $0.75 \mathrm{nS}$ \\
$K_{S K}$ & $0.4 \mu M$ \\
$E_{K}$ & $-90 \mathrm{mV}$ \\
$g_{U C L}$ & $1581 \mathrm{nS}$ \\
$k_{1}^{+}$ & $7.5 \cdot 10^{-7} \mu M^{-1} \mathrm{~ms}^{-1}$ \\
$k_{1}^{-}$ & $1.2 \mathrm{~ms}^{-1}$ \\
$k_{2}^{+}$ & $0.5 \mathrm{~ms}^{-1}$ \\
$k_{3}^{+}$ & $8.5 \cdot 10^{-5} \mathrm{~ms}^{-1}$ \\
$g_{D A P}$ & $0.462 \mathrm{nS}$ \\
$E_{N a}$ & $70 \mathrm{mV}$ \\
$\tau_{D A P}^{m}$ & $87 \mathrm{~ms}$ \\
$\tau_{D A P}^{h}$ & $860 \mathrm{~ms}$ \\
$n_{D A P} m$ & 2 \\
$K_{D A P m}$ & $0.09 \mu M$ \\
$A_{D A P}$ & 3.2 \\
$s_{D A P_{h}}$ & $0.025 \mu M$ \\
\hline
\end{tabular}

23

24

25

26

27

28

29

30

31

32

33

34

35

36

37

38

39

40

41

42

43

44

45

46

47

48

49

50

51

52

53

54

55

56

57

58

59

60

61

62

63

64

65 